\title{
Orientation splitting and its contribution to grain refinement during equal channel angular extrusion
}

\author{
Y. Huang $\cdot$ P. B. Prangnell
}

Received: 5 February 2008/Accepted: 28 March 2008/Published online: 26 July 2008

(C) Springer Science+Business Media, LLC 2008

\begin{abstract}
The early stage mechanisms of grain refinement during ECAE of a single-phase aluminium alloy have been studied using the EBSD technique. It was found that, in addition to the formation of shear-plane cell bands and shear bands by "simple shear", the development of deformation bands due to orientation splitting contributed significantly to the refinement of microstructure. "Regular" slab-like deformation bands and "irregular" transitional bands were observed after the first pass; both developed boundaries of high misorientations. In the second pass, moderate orientation splitting took place within the deformation bands, although new deformation bands were not detected. With increased strains, fine scale orientation splitting tended to occur in local bands, generating high densities of new high misorientation boundaries. The crystallographic features of the different types of orientation splitting are examined.
\end{abstract}

\section{Introduction}

Equal channel angular extrusion (ECAE) has emerged as a promising technique of severe plastic deformation $[1,2]$ and has been applied to produce ultra-fine grained or nanocrystalline structures in a range of metals and alloys $[2,3]$. Grain refinement by severe plastic deformation is mainly attributed to the development of strain-induced high misorientations via grain subdivision [4] and this is equally true in ECAE [2, 5]. It has been shown that grain

Y. Huang $(\varangle) \cdot$ P. B. Prangnell

Manchester Materials Science Centre, University of Manchester, Grosvenor Street, Manchester M17HS, UK

e-mail: yan.huang-2@manchester.ac.uk subdivision during ECAE takes place at various length scales [6]. In aluminium alloys, for example, during the early stages of deformation, dislocation cell blocks/bands subdivide the initial grains at the finest scale (order of one or two dislocation mean free paths) and shear bands form on top of the cell bands structure, whereas at a larger scale (near the grain size of the material) deformation bands often develop [6].

The contribution of deformation banding, via orientation splitting, to the generation of new high angle boundaries (HABs) during ECAE has been confirmed by several investigations [6-8]. However, its importance as one of the essential mechanisms of grain refinement seems not have been generally recognized. The present work was carried out to investigate the deformation structures and their evolution during the early stages of ECAE processing in a single-phase aluminium alloy, with emphasis on the formation and evolution of deformation bands and their contribution to grain refinement, in comparison to those from shear bands and pre-existing initial high angle boundaries.

\section{Experimental details}

A high purity $\mathrm{Al}-0.13 \mathrm{wt} \% \mathrm{Mg}$ alloy was used in this investigation. The material was cold rolled by $50 \%$ after casting and homogenization and recrystallized at $400{ }^{\circ} \mathrm{C}$ for $1 \mathrm{~h}$, giving a starting grain size of $\sim 300 \mu \mathrm{m}$ for ECAE. Extrusion billets (cylinders $15 \mathrm{~mm}$ in diameter and $100 \mathrm{~mm}$ in length) were machined out in the rolling direction, and subsequently processed by ECAE, via route A [1], at room temperature with a ram speed of $50 \mathrm{~mm} / \mathrm{min}$, to a total of three passes through a $90^{\circ}$ die lubricated by colloidal graphite. Figure 1 illustrates the ECAE process and 


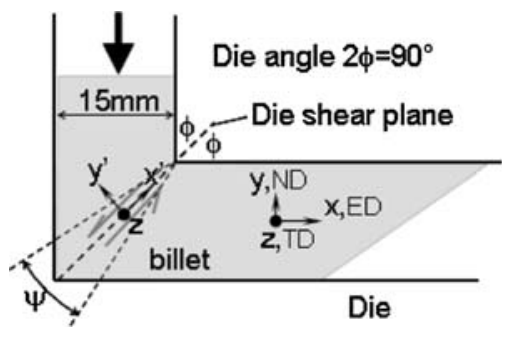

Fig. 1 Schematic diagram illustrating ECAE tooling, process parameters, and reference coordinates

reference coordinates. The deformation structures in the centre of the symmetrical plane of the ECAE processed billets were examined and characterized by electron backscattered imaging and electron backscattered diffraction (EBSD) in an FEI Siron field emission gun SEM (FEGSEM) equipped with a HKL Channel 5 acquisition system. In the data and EBSD maps presented, HABs defined as having misorientations $\geq 15^{\circ}$ are shown by black lines, and low angle boundaries (LABs) having misorientations of $<15^{\circ}$ are depicted by white lines. Due to misorientation noise, boundaries misoriented $<1.5^{\circ}$ were cut off.

\section{Results and discussion}

Cell bands and deformation bands formed in the first pass

It has been previously recognized that simple shear along the intersectional plane of the two extrusion channels (referred to hereafter as the die's shear plane) dominates during ECAE [1, 2, 9]. In the present experiment, cell bands, or cell blocks, comprised of dense dislocation walls, were found to form at an angle of $\sim 45^{\circ}$ to the extrusion direction (ED), and thus parallel to the die's shear plane, throughout the material after the first pass deformation, as shown in Fig. 2a. The EBSD map in Fig. $2 b$ shows the detailed structure of the cell bands and they are seen to be typically just one cell wide, which is consistent with previous observations [6]. A weaker second set of cell bands was also observed, as may be seen from Fig. 2b, which can be inferred to be associated with the slip roughly parallel to ED. The cell band structure was found to produce only limited grain subdivision and did not generate new HABs as the average misorientation across the cell bands was measured to be only $4.1^{\circ}$, although the cell width was close to $1 \mu \mathrm{m}$. However, the microstructure development was not uniform in the material, and deformation banding was found to occur in unstable grains via orientation splitting (Fig. 2a). The EBSD map in Fig. 3a shows a typical deformation structure developed in an unstable grain and it is seen that two types of deformation bands have formed. Primary deformation bands (e.g., A, B) characterized by well defined boundaries subdivided grains into slab morphology segments at an angle of $\sim 20^{\circ}$ to the extrusion direction (ED), with a width in the range of 20 $75 \mu \mathrm{m}$. Regions containing finer scale deformation bands, less-defined and irregular in morphology, were also seen, with wavy boundaries aligned more or less parallel to $\mathrm{ED}$ (e.g., area C). These irregular deformation bands had an average width of 7-30 $\mu \mathrm{m}$, although the width of individual bands varied along their length.

In the literature, deformation bands are typically reported as slab-like parallel sequences of volume elements with alternating average lattice orientation, as a result of the local simultaneous operation of fewer slip systems than would be required by homogeneous deformation, and terminate at grain boundaries [10]. However, it was found in the present experiments that many of the primary bands of a particular orientation ended inside a grain and the structure formed consisted of a "matrix" and embedded slab-like bands. This behavior may be explained by the limited dimensions of the deformation zone in ECAE. It is known that deformation banding involves orientation splitting at a scale close to the grain size and shear strain accommodation at the ends of bands requires an even larger scale. Such a scale requirement is not a problem in rolling or plain strain compression as the material yields on a far larger scale than the grain size under almost identical stress and strain states if friction is negligible. In contrast,
Fig. 2 (a) Backscatter electron image showing the deformation structure formed in the first pass and (b) EBSD map obtained from area $\mathrm{A}$ in (a) showing the fine scale cell band structure
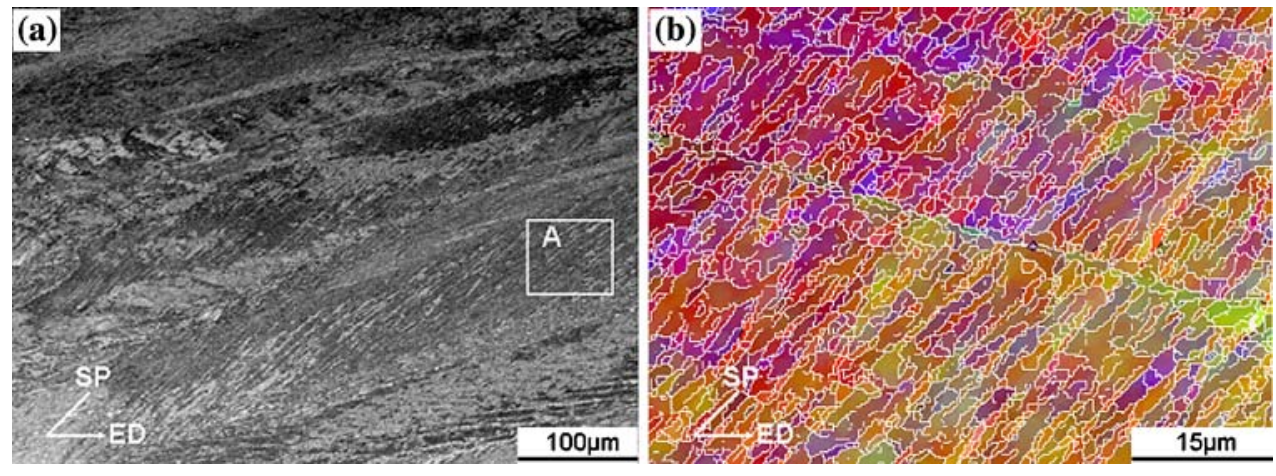


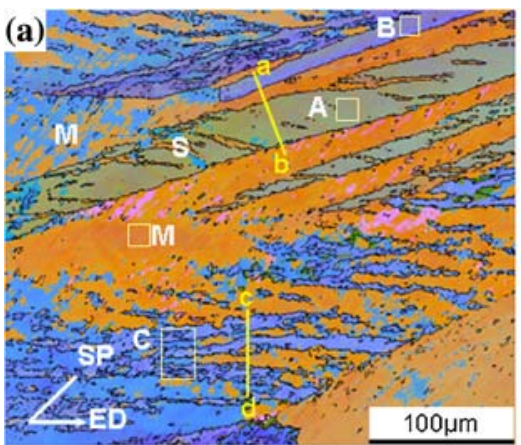

(c)

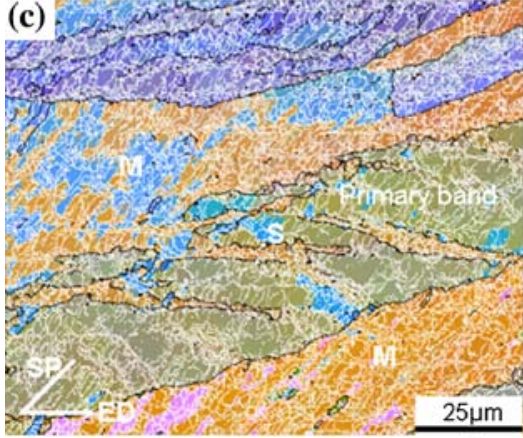

(d)

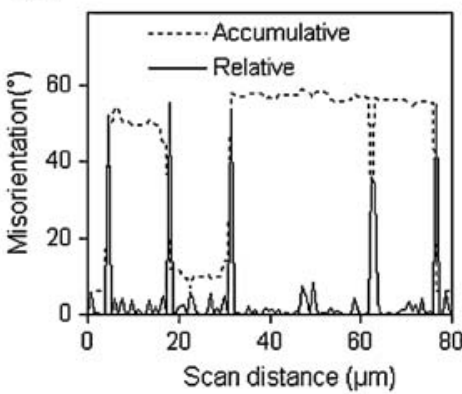

(b)

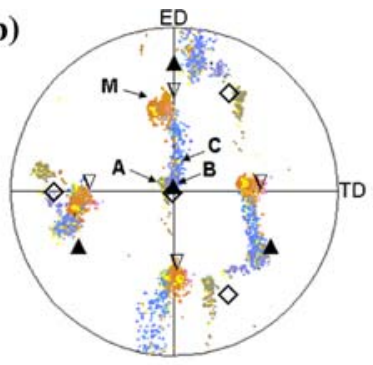

$\nabla-(81-1)<1-44>; \diamond-(111)[110] ; \Delta-(111)[-211]$ (e)

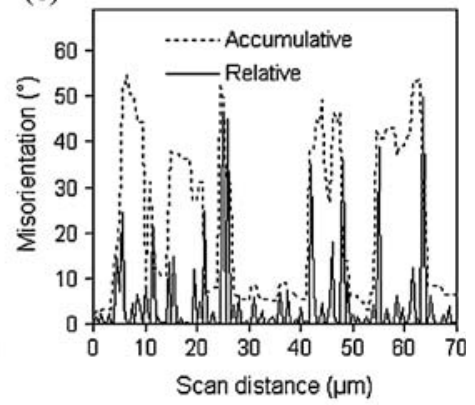

Fig. 3 (a) EBSD map showing a typical deformation band formed within an unstable grain in the first pass; (b) (111) pole figure showing the orientations and their spread (within the yellow spares) of the matrix (M), primary bands (A, B) and irregular bands (C) in (a); (c) the detailed structure of cell bands and secondary bands; and (d, e) linescans of boundary misorientations along $\mathrm{ab}$ and $\mathrm{cd}$ in (a) deformation in ECAE is concentrated in a narrow region along the die's shear plane. As a result, the simultaneous operation of reduced number of slip systems at a length scale of grain size can be difficult and bands terminated inside a grain may form.

The crystallographic features of both primary and irregular deformation bands and the associated matrix were examined by EBSD. It was found that while individual deformation bands developed various ultimate orientations, depending on their initial orientation and actual stress and strain conditions, most primary deformation bands were oriented close to recognized stable orientations in the ECAE reference frame. Figure $3 b$ shows the crystallographic orientations of the deformation bands $(\mathrm{A}, \mathrm{B}$, and $\mathrm{C})$ and the matrix $(\mathrm{M})$ in Fig. 3a in the ECAE reference frame (xyz or ED-ND-TD in Fig. 1). It is seen that the primary deformation bands $\mathrm{A}$ and $\mathrm{B}$ have developed an orientation of (111)[-110], which is stable in the ECAE reference frame but "random" in the simple shear reference frame with high miller indices of (25-1)[102]. On the other hand, the surrounding matrix was frequently found to be oriented near $(81-1)[1-44]$ in the ECAE reference frame or (111)[-1-12] in the simple shear reference frame $x^{\prime} y^{\prime} z$ (see Fig. 1), which is a major component of A-fibre in simple shear and allows the crystallographic slip plane to coincide with the die's shear plane. The orientation splitting related to the irregular deformation bands was relatively random and wide spread. However, in most cases, these bands were also found to evolve towards stable orientations. As shown in Fig. 3b, the irregular deformation bands in the area $\mathrm{C}$ in Fig. 3a have split towards an orientation close to (111)[-211] in one direction and into the orientation of the matrix, $(81-1)[1-44]$, at the other end in the ECAE reference frame (blue poles in Fig. 3b). Further orientation splitting within some primary deformation bands was observed, resulting in features typical of secondary deformation bands as horizontal intrusions into the primary bands (area S in Fig. 3a). Their orientations were found to lie between the characteristic orientation of the primary bands and the matrix.

According to the slip-line field approximation to ECAE by Segal [9], while deformation within a fan-shaped deformation zone with a relief angle of $\psi$ (see Fig. 1) is dominated by slip on the $\beta$ lines, approximately parallel to the die's shear plane, slip also occurs on $\alpha$ lines orthogonal to the $\beta$ lines, which is probably the cause of the second set of cell bands observed (Fig. 2b). The $\alpha$ slip shear strain generated can be estimated as $\gamma_{\alpha} \sim \psi(\mathrm{rad})$, which is small compared to the main shear strain on the $\beta$ lines (of $\sim 2 \cot \varphi$ ) [9]. At the outlet of deformation zone, the coordinate system for $\alpha$ slip coincides with the ECAE reference frame. The findings from crystallographic examination suggest that the deformation bands evolve towards a stable orientation corresponding to $\alpha$ slip, whereas 
the matrix tended to be oriented in coincidence with the $\beta$ slip in the die shear plane. The fact that the strain intensity of $\beta$ slip is significantly larger than that of $\alpha$ slip may explain why the volume of the "matrix" dominates in the banded structure. In a recent ECAE experiment on commercially pure aluminium by Zhilyaev et al. [8], it was shown that band-like features of deformation structure intensified with the increased die relief angle $(\psi)$, which is consistent with the present discussion, because both the dimension of the deformation zone and the intensity of $\alpha$ slip increase with $\psi$.

Cell bands approximately aligned with the die's shear plane also formed within the primary deformation bands but exhibited different features from those in the matrix. As shown in Fig. 3c, which is a fine EBSD map taken from the central area of Fig. 3a, the cell bands formed in the deformation bands are less-developed, more inhomogeneous and of substantially lower density than that in the matrix. The horizontal features in the structure, probably associated with the $\alpha$ slip, are seen to have further subdivided the primary bands into secondary bands, resulting in new HABs within the primary deformation band.

Contribution of deformation banding to the grain refinement in the first pass

While fine scale dislocation cell bands only introduced LABs in the first ECAE pass, deformation bands were found to be effective in creating high misorientations. In fact, deformation banding was the main mechanism responsible for the generation of new HABs during the first ECAE pass. EBSD analysis showed that most primary deformation bands developed boundaries with high misorientations and subdivided, together with the secondary deformation bands, the initial coarse grain, contributing directly to grain refinement. These slab-like bands usually exhibited alternating lattice orientations as may be seen from Fig. 3d, which shows misorientation variations across the boundaries of bands A and B in Fig. 3a. On the other hand, the regions comprised of finer scale irregular deformation bands exhibited a larger spread of orientation splitting and their individual boundaries were relatively less defined with a wide range of misorientations from 10 to $60^{\circ}$, as shown in Fig. 3e. Despite the fact that it was difficult to uniquely distinguish an area as being comprised of irregular deformation bands in many cases, it was still apparent, from extensive microstructural examination, that these regions made up a greater volume fraction of the deformation structure than primary deformation bands. To date, little attention has been paid to irregular deformation banding as a result of orientation splitting. This could be because their irregularity makes such bands difficult to quantify, or it is possible that they are a unique feature of orientation splitting in ECAE.
Table 1 Statistical parameters of first pass deformation bands (p-DBs, primary, i-DBs, irregular), and of shear bands (SBs) formed during the second pass

\begin{tabular}{llcll}
\hline & $\begin{array}{l}\text { Angle to } \\
\mathrm{ED}\left({ }^{\circ}\right)\end{array}$ & $\begin{array}{l}\text { Width } \\
(\mu \mathrm{m})\end{array}$ & $\begin{array}{l}\text { Misorientation } \\
\left({ }^{\circ}\right)\end{array}$ & $\begin{array}{l}\text { Spacing } \\
(\mu \mathrm{m})\end{array}$ \\
\hline p-DBs & $15-25$ & $20-75$ & $\sim 30-50$ & \\
i-DBs & $0-15$ & $5-15$ & $\sim 10-60$ & \\
SBs & $45 \pm 5$ & $0.8-8$ & $\sim 5-30$ & $5-25$ \\
\hline
\end{tabular}

Although it was difficult to accurately quantify the contribution of deformation banding to grain refinement, as their distribution was not uniform and most irregular deformation bands contained only less defined HABs segments, EBSD (mean linear intercept) analysis over an area that covered about 100 initial grains showed that the HABs generated by deformation banding in the first pass refined the material from the initial grain size of $300 \mu \mathrm{m}$ into a deformed structure with an equivalent grain size of $\sim 15 \mu \mathrm{m}$. The microstructural parameters of the deformation bands estimated from EBSD measurements are given in Table 1.

Grain subdivision in the second pass

The backscattered image in Fig. 4 shows a typical deformation structure formed after the second ECAE pass. Shear bands, parallel to the die shear plane ( $\sim 45^{\circ}$ to ED), have now formed throughout the microstructure and the deformation bands formed in the first pass are compressed and elongated. In situ FEGSEM examination with the help of tilting the specimen and EBSD measurements confirmed that the deformation bands seen in Fig. 4, such as D and E, were formed in the first pass, rather than in the second pass. They were found to have undergone heavy shear banding across their boundaries, which would not occur if the deformation bands had formed in the second pass.

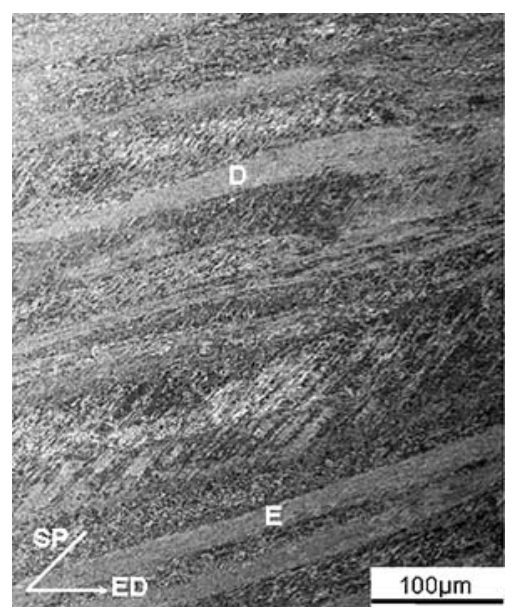

Fig. 4 Backscatter electron image showing the deformation structure formed after the second pass 

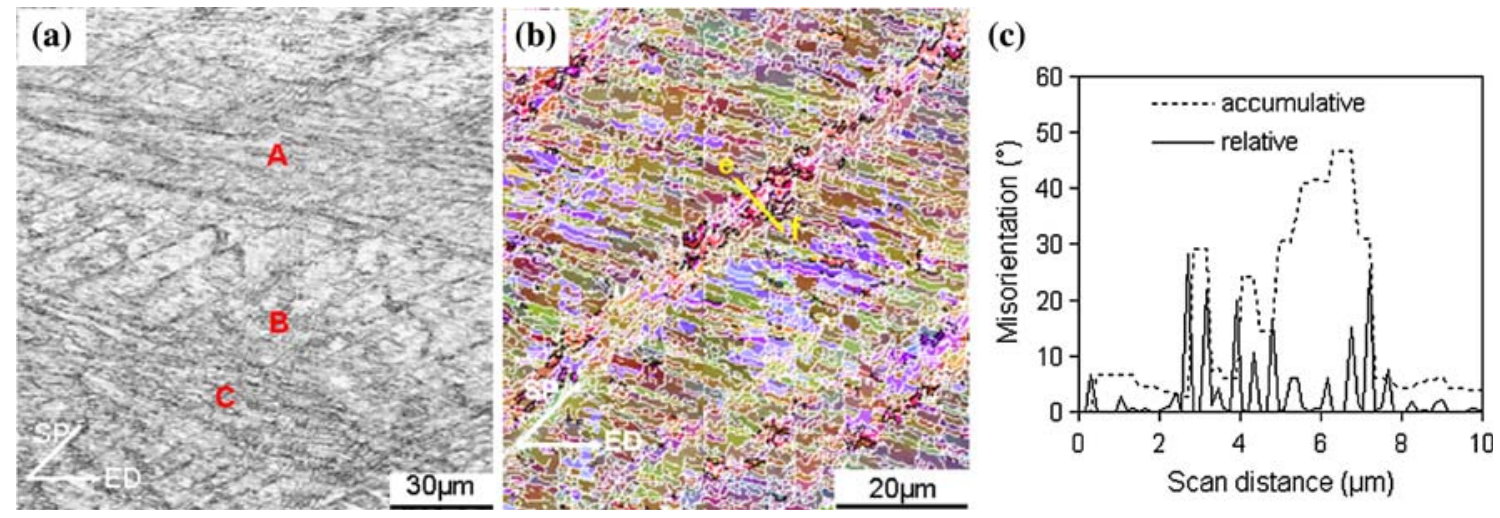

Fig. 5 (a, b) EBSD maps obtained from the deformation zone showing the variation of shear bands intensity from grain to grain and detailed structures of shear bands, respectively; (c) misorientation linescan along ef in (b)

Furthermore, all the deformation bands had evolved to align with the overall grain elongation direction in response to the total shear strain applied after the second pass, as shown in Fig. 4, which confirms that these features were present prior to the second pass deformation. In fact, no evidence of new deformation bands being formed was observed and in the second pass grain subdivision was mainly caused by the introduction of shear bands, as well as from an increase in boundary area and misorientation of the first pass deformation bands with strain, particularly, between the irregular deformation bands.

Figure 5a is an EBSD band contrast quality map obtained from the deformation zone in a specimen extruded halfway through its second pass, showing essential features of the shear banding. It can be seen that the shear bands cut through the aligned cell bands formed in the first pass and dominate the deformation structure. It is also seen that the intensity of shear bands varies from grain to grain (A and B, Fig. 5a). EBSD analysis revealed that grains oriented near the ideally crystallographically correlated orientation (111)[-1-12] in the simple shear reference frame exhibited more intense shear banding than the randomly oriented grains (e.g., grains $\mathrm{A}$ and $\mathrm{B}$ in Fig. 5a). In regions that contained first pass irregular deformation bands (area C), shear banding seemed to have been largely restricted due to the existence of a high density of previously formed HABs. Figure $5 \mathrm{~b}$ shows the detailed structural features of shear bands and the typical misorientations generated across them are given in Fig. 5c. The microstructural parameters of the shear bands determined from EBSD measurements are given in Table 1. Regardless of crystallographic orientations of individual grains, the shear bands showed common features: (i) all were substantially parallel to the die's shear plane; (ii) the crystal lattice within the shear bands was rotated with respect to the matrix (see the "accumulative misorientation" plot in Fig. 5c); and (iii) the material inside most shear bands was not uniformly sheared and instead the shear bands were composed of regularly spaced bundles of micro shearbands, each carrying intense strains. As a result, misorientations in the range of $5-30^{\circ}$ were created across the individual layers within the bands and the boundaries between the shear bands and the matrix. However, the majority of the misorientations across the shear bands were below $15^{\circ}$ and shear banding only generated a limited density of HAB segments. The EBSD map in Fig. 6a shows HABs in the deformation structure formed after the second pass and it is seen that most HABs are associated with the deformation bands formed in the first pass.

While in the second pass the first pass primary deformation bands were essentially compressed and elongated, resulting in an increase in $\mathrm{HAB}$ area and finer average $\mathrm{HAB}$ spacing, the regions containing irregular deformation bands often evolved into areas of high HAB density (e.g., area $\mathrm{F}$ in Fig. 6a). It was found that most irregular deformation bands, which were only bordered by HAB segments after formation in the first pass, developed straighter, more continuous, HABs with increased misorientations during the second pass, as shown in Fig. 6b. EBSD analysis revealed that the effective mean $\mathrm{HAB}$ spacing in these areas was often as small as only about $2 \mu \mathrm{m}$, although the overall distribution of HABs was not uniform.

\section{Deformation structure formed in the third pass}

In the third pass, no new deformation banding was seen and shear banding was far weaker than in the second pass so that few new HABs were found to be generated. However, most boundaries of previously formed deformation bands and shear bands developed higher misorientations and they were compressed during deformation towards ED, resulting in the formation of a fibre structure, as shown in Fig. 7. The HAB fraction and spacing in the fibre structure was measured to be $\sim 30 \%$ and $\sim 1.65 \mu \mathrm{m}$, respectively. Although it was difficult to distinguish the contributions of individual types of HABs to grain subdivision, calculations according to the 
Fig. 6 EBSD maps showing (a) the general $\mathrm{HAB}$ structure formed in the third pass and (b) the HAB structure evolved from irregular deformation bands
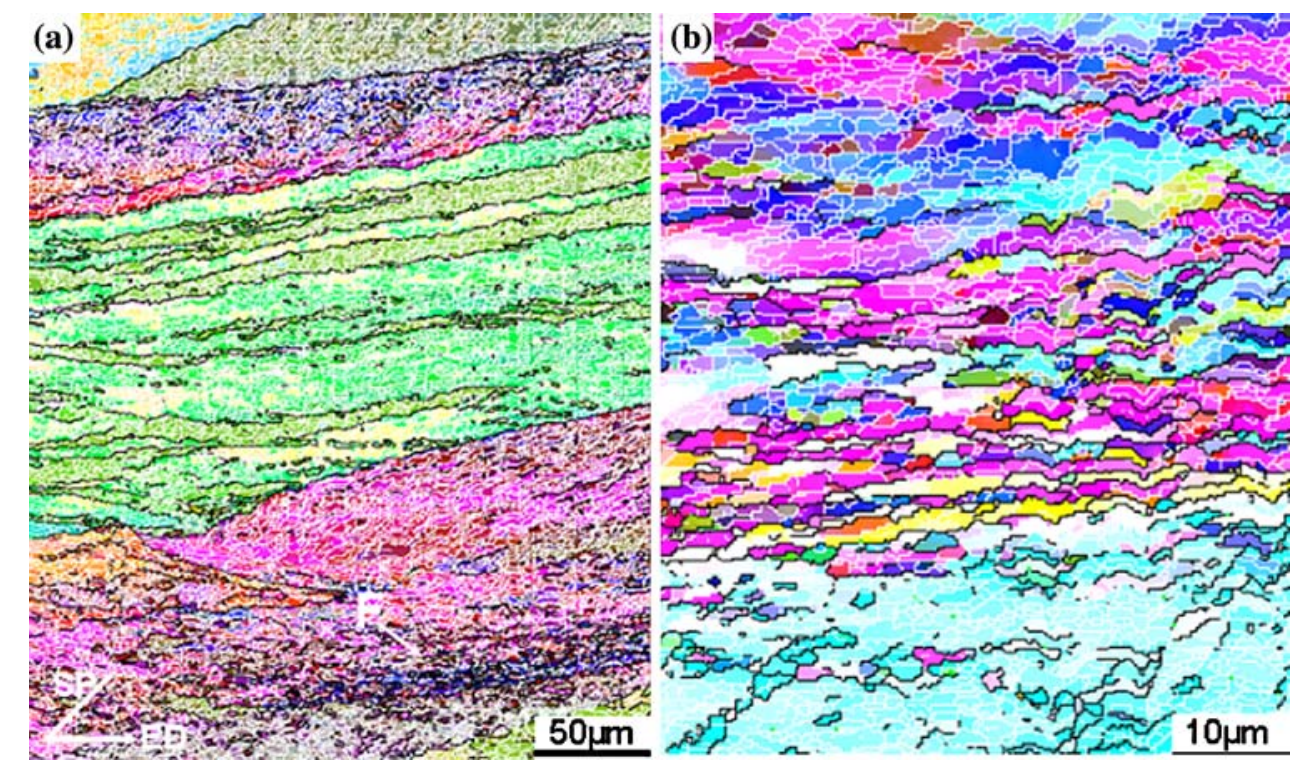

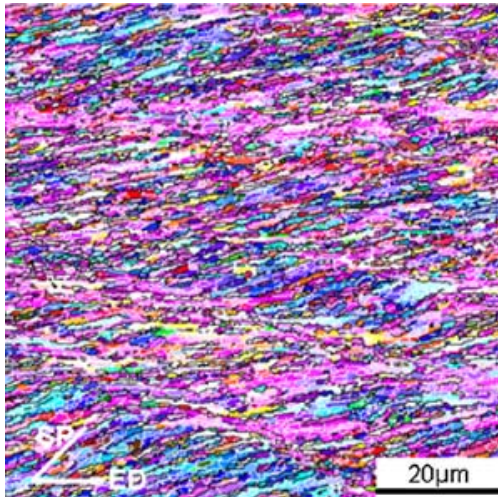

Fig. 7 EBSD map showing the fibre structure formed after the third ECAE pass

effect of geometrical compression [9] showed that, after the third pass, the first pass deformation bands should have approximately produced an average HAB spacing of $3.6 \mu \mathrm{m}$. In comparison, the equivalent mean HAB spacing from the shear bands formed in the second pass was about $6.5 \mu \mathrm{m}$, assuming that their boundaries all evolved into high-angle misorientations. Thus, the HABs that existed prior to the third pass could have produced a mean HAB spacing of $\sim 2.3$ $\mu \mathrm{m}$ without the formation of any new HABs. In contrast, geometrical compression of the original grain structure with no grain subdivision would lead to a HAB spacing of $\sim 50 \mu \mathrm{m}$ by the third pass. Although these figures are only rough estimates, the large discrepancy with the measured value of $\sim 1.65 \mu \mathrm{m}$ suggests the occurrence of other mechanisms responsible for the generation of new HABs in the third pass. In fact, the uniformly distributed HABs obtained in Fig. 7 are unlikely to have evolved from the heterogeneous structures formed in the previous passes without further grain subdivision.
Figure 8a is an EBSD map obtained at the beginning of the third pass deformation in an area free of deformation bands, showing that new HABs segments are formed in line with the first pass cell bands structure. The associated orientation spread of up to $45^{\circ}$ within an area of $\sim 20 \times 20$ $\mu \mathrm{m}^{2}$ is shown in Fig. 8b. Such a large orientation spread with the involvement of new HABs generation was not observed in the previous pass outside of deformation bands and shear bands and cannot be explained neither by normal shearing in the die shear plane nor by the gradual increase in misorientation across cell bands through absorbing dislocations [11]. Figure 8c shows a misorientation linescan across the structure in Fig. 8a (along gh) and it is seen that the domains separated by the newly formed HABs exhibit alternate orientations and in each domain the orientation remains nearly constant. This behavior is similar to that of conventional coarser scale orientation splitting in deformation bands (see Fig. 3d, e) rather than that of orientation development in shear bands (see Fig. 5c), in which the maximum orientation spread is the result of the accumulative lattice rotation towards one direction, and suggests that this new fine scale grain subdivision is likely to be due to local orientation splitting. According to model simulations (P. Bate, private communication), more localized orientation splitting can potentially occur in simple shear, compared to in-plane strain compression, because of the high rate of material spin, resulting in more rapid deviations in spin upon perturbations in stress, or orientation.

\section{Summary}

Deformation structures and their evolution during the early stages of ECAE processing in an $\mathrm{Al}-0.13 \mathrm{Mg}$ alloy have 

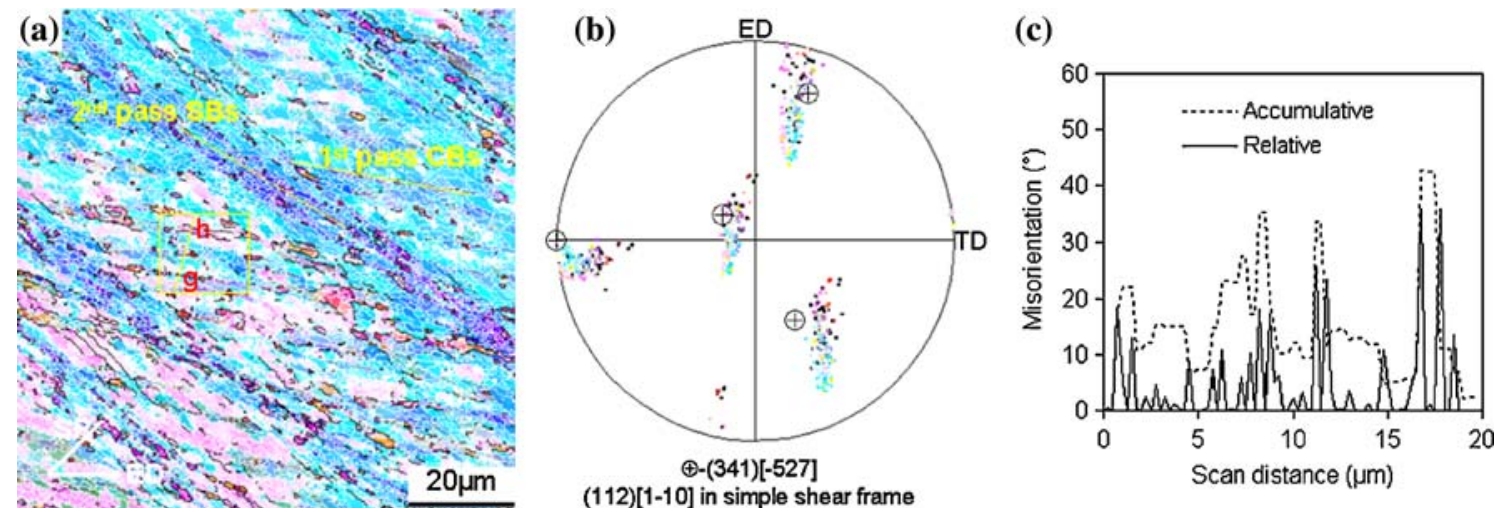

Fig. 8 (a) EBSD map showing the development of new HABs in the early stages of third pass deformation; (b) 111 pole figure showing the orientation spread within the yellow square in (a); and (c) misorientation linescan along gh in (a)

been investigated. Deformation banding due to orientation splitting was found to be the main mechanism responsible for the generation of new HABs in the first pass. Irregular deformation bands were found to be a more general feature of the deformation structure than "regular" slab-like or primary deformation bands. Both types of deformation bands tended to rotate to stable crystallographic orientations and developed boundaries of high misorientations upon formation, which evolved with strain and constituted a significant proportion of HABs in the highly misoriented fibre structure formed after the third pass. In comparison, fine scale shear plane aligned dislocation cell bands formed in the first pass only generated low misorientations. Shear bands dominated the second pass deformation structure and created HAB segments of medium misorientations. The generation of new HABs in the third pass was probably due to localized orientation splitting, although more experimental evidence and theoretical analysis are needed to confirm this.

Acknowledgements The authors would like to acknowledge the financial support of the University of Manchester EPSRC Light Alloys Portfolio Partnership (EP/D029201/1) for this project.

\section{References}

1. Segal VM (1995) Mater Sci Eng A 197:157. doi:10.1016/09215093(95)09705-8

2. Valiev RZ, Islamgaliev RK, Alexandrov IV (2000) Prog Mater Sci 45:103. doi:10.1016/S0079-6425(99)00007-9

3. Wang J, Iwahashi Y, Horita Z, Furukawa M, Nemoto M, Valiev RZ, Langdon TG (1996) Acta Mater 44:2973. doi:10.1016/13596454(95)00395-9

4. Hansen N, Huang X, Hughes DA (2001) Mater Sci Eng A 386:3. doi:10.1016/S0921-5093(01)01191-1

5. Zhu YT, Lowe TC (2000) Mater Sci Eng A 291:46. doi:10.1016/ S0921-5093(00)00978-3

6. Prangnell PB, Bowen JR, Apps PJ (2004) Mater Sci Eng A 375377:178. doi:10.1016/j.msea.2003.10.170

7. Werenskiold JC, Roven HJ (2005) Mater Sci Eng A 410-411: 174. doi:10.1016/j.msea.2005.08.049

8. Zhilyaev AP, Oh-ishi K, Raab GI, McNelley TR (2006) Mater Sci Eng A 441:245. doi:10.1016/j.msea.2006.08.029

9. Segal VM (1999) Mater Sci Eng A 271:322. doi:10.1016/S09215093(99)00248-8

10. Kuhlman-Wilsdorf D (1999) Acta Mater 47:1697. doi:10.1016/ S1359-6454(98)00413-3

11. Hurley PJ, Humphreys FJ (2003) Acta Mater 51:1087. doi:10.1016/ S1359-6454(02)00513-X 